\title{
A radiological classification system for talocalcaneal coalition based on a multi-planar imaging study using CT and MRI
}

\author{
Sanghyeok Lim $\cdot$ Hyeon Kyeong Lee $\cdot$ Sooho Bae $\cdot$ \\ Nae-jung Rim • Jaeho Cho
}

Received: 1 February 2013 /Revised: 17 June 2013 / Accepted: 20 June 2013 /Published online: 24 July 2013

(C) The Author(s) 2013. This article is published with open access at Springerlink.com

\begin{abstract}
Objective To develop a radiological classification system for talocalcaneal coalition suitable for adults.

Methods and materials A retrospective review was performed on patients diagnosed with talocalcaneal coalition from July 2001 to November 2011. Based on the cartilaginous or bony nature, facet joint orientation and bony structure morphology, we classified talocalcaneal coalitions into four types: I (linear with or without posterior hooking), II (talar overgrowth), III (calcaneal overgrowth) and IV (complete osseous).

Results Seventy feet (59 patients) with talocalcaneal coalition were evaluated by CT (61/70 feet) using multi-planar reformation and/or magnetic resonance imaging (43/70 feet). Type I, II, III and IV coalitions were detected in 45 (64\%), $10(14 \%), 13(19 \%)$, and 2 feet (3\%), respectively. Fracture fragments were observed in 16 feet (seven Type I and nine Type III coalitions) with hooked or overgrown calcanei and in one foot in the talus (Type I). Eleven patients had bilateral talocalcaneal coalitions; ten patients had coalitions of the same type and one had both Type I and Type III coalitions. Among 48 patients with unilateral involvement, the left and right feet were affected in 26 and 22 patients, respectively. Conclusions A classification system for talocalcaneal coalition based on multi-planar imaging studies was developed.
\end{abstract}

S. Lim $\cdot$ H. K. Lee $(\triangle) \cdot$ S. Bae $\cdot$ N.-j. Rim

Department of Radiology, Armed Forces Capital Hospital,

San13-4, Yul-dong, Bundang-gu, Seongnam-si,

Gyeonggi-do 463-040, Korea

e-mail: hyeonkyeongleemd@gmail.com

J. Cho

Department of Orthopedics, Armed Forces Capital Hospital,

San13-4, Yul-dong, Bundang-gu, Seongnam-si,

Gyeonggi-do 463-040, Korea
Key Points

- A classification system for talocalcaneal coalition based on multi-planar imaging was developed.

- The relative frequencies of different talocalcaneal coalition types were determined.

- Fracture fragments were easily distinguished and frequent$l y$ originated from the calcaneus.

- Fracture fragments were mostly associated with Type I (linear) with posterior hooking and Type III (calcaneal overgrowth).

Keywords Classification · Anatomy · Talocalcaneal coalition syndrome $\cdot \mathrm{X}$-ray computed tomography $\cdot$ Magnetic resonance imaging

\section{Introduction}

Tarsal coalition is defined as an abnormal union between two or more tarsal bones and is recognised as a common cause of rigid flatfeet. Tarsal coalition may be fibrous (synfibrosis or syndesmosis), cartilaginous (synchondrosis) or osseous (synostosis) in nature. The two most common coalitions are talocalcaneal and calcaneonavicular coalitions. The cause of tarsal coalition is reported to be a failure of segmentation of the primitive mesenchyme during development [1]. Tarsal coalitions are clinically significant because they prevent normal joint motion, and they are frequently associated with non-traumatic pain and cavus foot deformity [2-6].

After the anatomical nature of talocalcaneal coalition was reported [7], its radiographic signs and findings were described in multiple reports [8-10]. The introduction of computed tomography (CT) and magnetic resonance imaging (MRI) improved the understanding of the anatomic properties and characteristics of talocalcaneal coalition [11-19]. Based on three-dimensional (3D) reconstructions, one report 
classified talocalcaneal coalition of children into five types (linear, linear with a posterior hook, shingled, complete osseous and posterior) and attempted to provide helpful information for surgical planning [20]. However, in our experience with adult patients, there are many cases of talocalcaneal coalition with superiorly overgrown calcanei covering the calcaneal articular facet of the talus that could not be classified into any of these five types. Therefore, in this study, we devised a new radiological classification system for talocalcaneal coalition encompassing cases of talocalcaneal coalition with a superiorly overgrown calcaneus covering the calcaneal articular facet of the talus.

\section{Materials and methods}

After obtaining approval from the Institutional Review Board, we retrospectively collected cases by searching the clinical database using the search terms of "coalition", "tarsal coalition" and "talocalcaneal coalition". A total of 77 patients were identified from July 2001 to November 2011. All of the patients were soldiers or officers in the Korean military. Of these patients, data from CT or MRI, or both types of imaging, were available on our picture archiving communication system (PACS) for 65 patients. Two experienced radiologists (H.K.L. with 24 years of experience and S.L. with 4 years of experience) reviewed CT and MR images and identified 59 patients with talocalcaneal coalition. The remaining six patients diagnosed with calcaneonavicular coalition were excluded.

As defined by the ankle CT protocol at our institution, all patients were positioned supine on the examination table with the bottom of the affected foot placed against a positioning box. Each affected foot was scanned from the bottom proximally through the ankle joint using one of the following CT machines: MX8000 (Philips Medical Systems, Best, The Netherlands), SOMATOM Sensation 4 (Siemens Healthcare, Forchheim, Germany) or Brilliance 64 (Philips Medical Systems). All examinations were performed without intravenous contrast enhancement. Axial, direct coronal and sagittal reformatted images were obtained in cases before the 64channel CT machine equipped with volume-rendering software was available. In cases after the 64-channel CT machine became available, the raw axial 2D CT data were uploaded to a workstation, and volume-rendering software was used to reconstruct the sagittal and coronal planes using a standard algorithm.

As defined by the ankle MRI protocol at our institution, all patients were positioned supine on the examination table within the magnetic bore. We obtained five series of MR images, including coronal fast spin echo (FSE) T2-weighted images with fat suppression (TR/TE, 2,816/96 ms), sagittal SE T1-weighted images (TR/TE, 527/20 ms), sagittal FSE T2weighted images with fat suppression (TR/TE, 2,816/96 ms), axial SE T1-weighted images (TR/TE, 414/40 ms), and axial FSE T2-weighted images with fat suppression (TR/TE, 4,022/96 ms), using MRI machines (MAGNETOM Symphony 1.5 T; Siemens Healthcare/Signa Excite 1.5 T; GE Healthcare, Milwaukee, WI, USA).

After all of the images had been reviewed by two radiologists and a consensus was achieved, the cases were classified as Type I, II, III or IV coalition (Fig. 1) as follows: Type I, linear, parallel, or nearly parallel to the adjacent subtalar
Fig. 1 Talocalcaneal coalition on coronal $\mathrm{CT}$ images $(\mathbf{a}, \mathbf{c}, \mathbf{e}$ and $\mathbf{g})$ and coronal fat-suppressed T2weighted images (TR/TE, 2,816/ $96 \mathrm{~ms})(\mathbf{b}, \mathbf{d}, \mathbf{f}$ and $\mathbf{h})$. a, b Type I (linear) talocalcaneal coalition: parallel or nearly parallel to the adjacent subtalar joint on the same plane. The arrows indicate the coalition. c, $\mathbf{d}$ Type II (talar overgrowth) talocalcaneal coalition: inferiorly overgrown talus covering the talar articular facet of the calcaneus. The arrows indicate the coalition. e, f Type III (calcaneal overgrowth)

talocalcaneal coalition: superiorly overgrown calcaneus covering the calcaneal articular facet of the talus. The arrows indicate the coalition. g, h Type IV (complete osseous) talocalcaneal coalition.

The arrows indicate the coalition
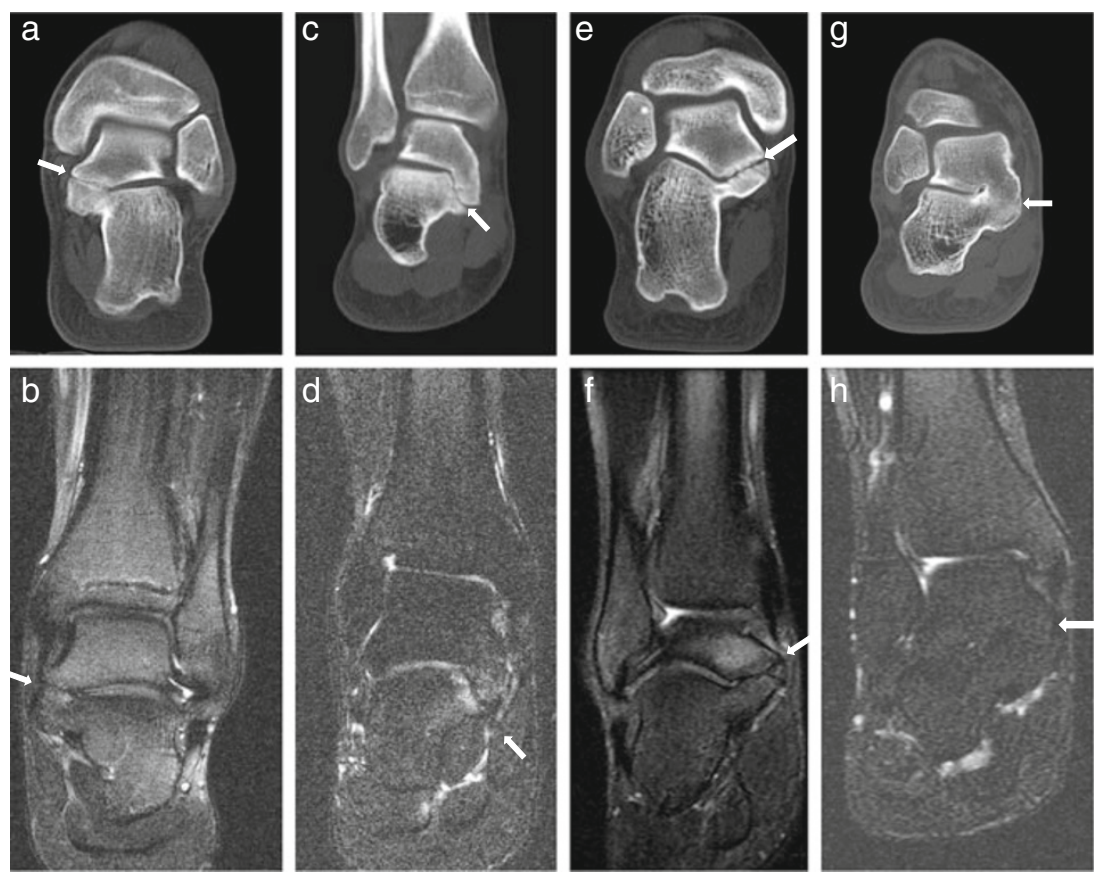

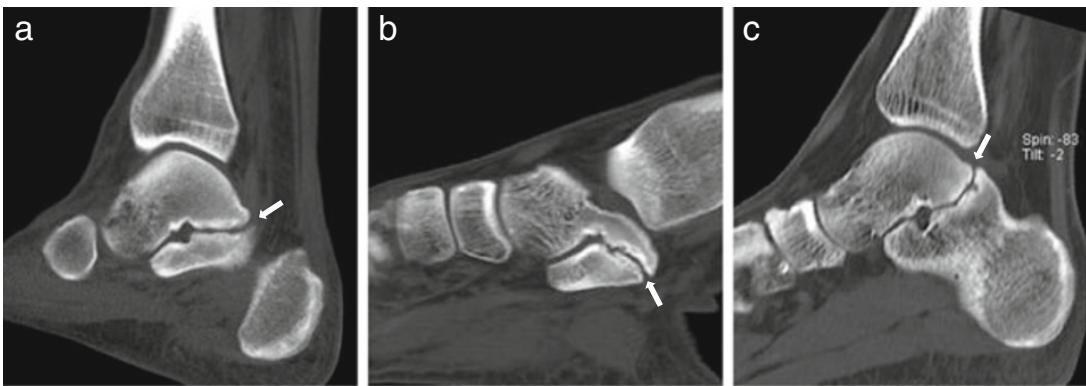

Fig. 2 Type I talocalcaneal coalition with or without posterior hooking on sagittal CT images. a A 23-year-old man with Type I talocalcaneal coalition without posterior hooking. The arrow indicates the coalition. b A 23-year-old man with Type I talocalcaneal coalition with posterior

joint on the same plane, with or without posterior hooking (Fig. 2); Type II, inferiorly overgrown talus covering the talar articular facet of the calcaneus; Type III, superiorly overgrown calcaneus covering the calcaneal articular facet of the talus; Type IV, complete osseous. We also evaluated the presence of fracture fragments and their origin. The origin of fracture fragments was determined by the surface margin, displacement and signal changes on CT and MRI (Figs. 3 and 4).

To compare fracture sites between the talus and calcaneus, a chi-squared test was used. In statistical comparisons, $p$ values less than 0.05 were considered statistically significant. Statistical analysis was performed using statistical software (Excel; Microsoft Corporation, Redmond, WA, USA).

\section{Results}

Among 59 patients diagnosed as talocalcaneal coalition, 11 patients $(19 \%)$ had bilateral coalitions. In ten patients, the bilateral coalitions were of the same type, and one patient had Type I and Type III coalitions. Among the remaining 48 patients $(71 \%)$ with unilateral involvement, the left and right feet were affected in 26 and 22 patients, respectively. For talar hooking. The arrow indicates the coalition. c A 24-year-old man with Type I talocalcaneal coalition with posterior calcaneal hooking. The arrow indicates the coalition

these 70 feet, 45 (64\%), 10 (14\%), $13(19 \%)$ and $2(3 \%)$ coalitions were classified as Type I, II, III and IV coalitions, respectively (Table 1).

Most of the coalitions ( $90 \%, 63 / 70$ feet) involved the middle talocalcaneal joint. Four and two feet displayed coalition at the middle/posterior and posterior joints, respectively, and all of these coalitions were Type I coalitions. A Type IV coalition involving the anterior/middle joint was detected in one foot.

Fracture fragments were found in 17 feet $(24 \%, 17 / 70$ feet); eight Type I and nine Type III coalitions. The fragments more frequently originated from the calcaneus (16 feet; $p<0.05$ ). All of the fractures appeared to occur in overgrown or hooked bone. A fragment in one foot that originated from the talus was classified as Type I.

Bone marrow oedema at both bones was evident in 37 ( $86 \%)$ of 43 feet with MRI data. In five feet (12\%, 5/45 feet) of three Type I and two Type III coalitions, bone marrow oedema was equivocal at both bones; in one type I foot ( $2 \%$, $1 / 45$ feet), bone marrow oedema was evident in the calcaneus but equivocal in the talus. Clinically, pain at rest that increased with athletic activity was the major symptom and was reported in 60 (86\%) of 70 feet. Among the ten feet without clinical symptoms, seven Type I, one Type II and two Type III coalitions were observed.
Fig. 3 A 27-year-old man with Type III talocalcaneal coalition with a fracture fragment originating from the calcaneus. The CT (a) and coronal fatsuppressed $\mathrm{T} 2$ weighted (TR/TE, 2,816/96 ms) (b) images show the fracture line (arrows), articular changes and bone oedema
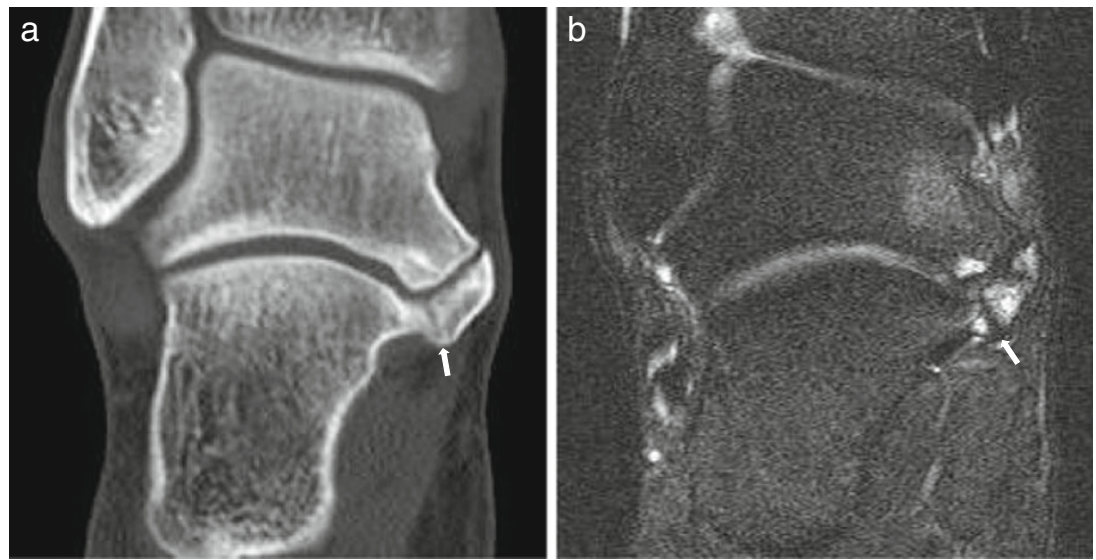
Fig. 4 A 29-year-old man with Type I talocalcaneal coalition with a fracture fragment originating from the calcaneus. The CT (a) and coronal fatsuppressed T2 weighted (b) (TR/TE, 2,816/96 ms) images show a facture line (arrow) and fracture fragment (arrowhead) from the calcaneus. The MR images (b) show marrow signal changes (dashed arrows) at the articular surface of the talocaneal coalition
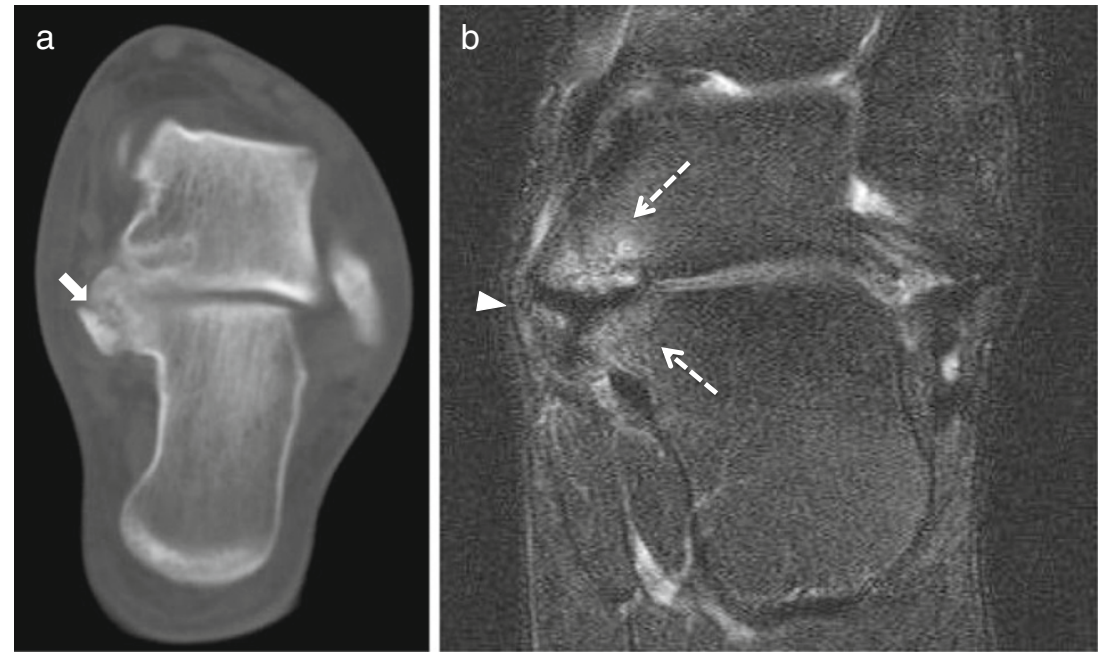

Posterior talar hooking and posterior calcaneal hooking were detected in 25 and 5 feet with Type I coalitions, respectively. All five feet with posterior calcaneal hooking involved the posterior talocalcaneal joint, and the middle joint was also involved in three of these feet.

\section{Discussion}

Until the early 1980s, talocalcaneal coalition was diagnosed using standard X-ray, which is still commonly used for screening purposes. However, radiographic confirmation of talocalcaneal coalition was more difficult than for fusion at other locations [21]. Additionally, in the present study, only $23 \%$ of the coalitions could be diagnosed by X-ray. Even in those cases, either subtyping or detection of fracture was not possible.

Since the introduction of CT and MRI, both imaging modalities have been used to differentiate osseous from non-osseous coalition and depict the extent of joint involvement and secondary degenerative changes, which are vitally important for surgical planning. Short inversion time recovery MRI may reveal bone marrow oedema along the margins of abnormal articulation, an important clue for the diagnosis

Table 1 A radiological classification system for adult talocalcaneal coalition based on a multi-planar imaging study using CT and MRI

\begin{tabular}{|c|c|c|}
\hline \multicolumn{2}{|c|}{ Type } & No. of feet (percentage) \\
\hline I & Linear with or without posterior hooking & $45(65 \%)$ \\
\hline II & Talar overgrowth & $10(14 \%)$ \\
\hline III & Calcaneal overgrowth & $13(19 \%)$ \\
\hline IV & Complete osseous & $2(3 \%)$ \\
\hline
\end{tabular}

$[17,21,22]$. Frequently, CT or MRI is required to confirm the diagnosis of talocalcaneal coalition when radiographic findings are equivocal. Occasionally, tarsal coalition may be encountered incidentally during the evaluation of adolescent feet for other indications [19].

In this study, we analysed the characteristics of talocalcaneal coalition among 70 feet, which is the largest collection to our knowledge. The frequency of complete osseous coalition was lower than expected, particularly in comparison with a previous report based on adolescent patients [20]. In addition, we did not find the peripheral posterior coalition reported previously [20]. We suggest two reasons for these discrepancies. First, during the health-screening process for military recruitment, subjects with complete osseous coalition are more likely to have been excluded because it is a disadvantageous factor. Second, in adults, complete or peripheral posterior osseous coalition is typically asymptomatic. Thus, a subject with complete osseous coalition would be less likely to visit a hospital.

It is worth noting that most of the cases involved the middle talocalcaneal joint. Only two cases involved the posterior joint instead of the middle talocalcaneal joint, and both of these cases involved Type I coalitions. Four additional Type I cases exhibited coalition with middle/posterior involvement. All Type II and III coalitions involved only the middle talocalcaneal joint.

The probability of associated fractures in talocalcaneal coalition has not been reported previously. In this study, fracture fragments were detected at a frequency of one out of four cases. Most of these originated from the calcaneus, and all of these cases involved either Type I coalitions with posterior calcaneal hooking or Type III coalitions. A fragment originating from the talus was detected in one Type I coalition with posterior talar hooking. Fracture fragments were significantly more likely to originate from the 
calcaneus than from the talus $(p<0.05)$. In most cases, the origins of fracture fragments were easily distinguished by evaluating either CT or MRI findings. In particular, the margin changes, direction of displacement and/or signal changes on MRI were extremely helpful (Fig. 4). Because there is a chance that the fracture fragments cause symptoms, it is advantageous to report their presence so that surgeons can consider their removal. In addition, in Type I with posterior calcaneal hooking or a Type III coalition, the presence of fracture fragments should be monitored more carefully.

Bilateral coalitions were found only in 11 of 59 patients (19\%), which was much lower than the findings of a previous report $(63 \%)[20]$. This likely originated from the selection bias of our study. This study only included members of the Korean military, which has a pre-screening system for the recruitment of candidates for officers and soldiers.

While evaluating the cases enrolled in this study, we developed an efficient scheme for classifying and describing talocalcaneal coalition. With coronal images, the type of coalition can be determined. Then, the presence of talar or calcaneal hooking can be detected on sagittal images. The presence of fractures and their origin can be detected using either $\mathrm{CT}$ or MRI. This classification system provides information regarding the incision site and approach of resection to surgeons. Additionally, fracture fragments would be more thoroughly searched in Type I with posterior calcaneal hooking and Type III coalitions. The limitation of the present study lies in its retrospective nature. Moreover, the present study is a singlecentre study including only male soldiers and officers.

In conclusion, we established a classification system for talocalcaneal coalition suitable for adult patients and report that the probability of associated fractures is $24 \%$. The fractures mostly originated from the calcaneus and were associated with Type I coalition with posterior hooking or Type III.

\section{Conflicts of interest None.}

Open Access This article is distributed under the terms of the Creative Commons Attribution License which permits any use, distribution, and reproduction in any medium, provided the original author(s) and the source are credited.

\section{References}

1. Jack EA (1954) Bone anomalies of the tarsus in relation to peroneal spastic flat foot. J Bone Joint Surg Br 36B(4):530-542
2. Stuecker RD, Bennett JT (1993) Tarsal coalition presenting as a pes cavo-varus deformity: report of three cases and review of the literature. Foot Ankle 14(9):540-544

3. Knapp HP, Tavakoli M, Levitz SJ, Sobel E (1998) Tarsal coalition in an adult with cavovarus feet. J Am Podiatr Med Assoc 88(6):295-300

4. Varner KE, Michelson JD (2000) Tarsal coalition in adults. Foot Ankle Int 21(8):669-672

5. Barrett SE, Johnson JE (2004) Progressive bilateral cavovarus deformity: an unusual presentation of calcaneonavicular tarsal coalition. Am J Orthop (Belle Mead NJ) 33(5):239-242

6. Julia RC, Kristina MK (2004) Radiographic diagnosis of tarsal coalition. Am J Roentgenol 182(2):323-328

7. Zuckerkandl E (1877) Ueber einen fall von synostose zwischen talus und calcaneus. Allg Weiner Med Ztg 22:293-294

8. Vaughan WH, Segal G (1953) Tarsal coalition, with special reference to roentgenographic interpretation. Radiology 60(6):855-863

9. Conway JJ, Cowell HR (1969) Tarsal coalition: clinical significance and roentgenographic demonstration. Radiology 92(4):799-811

10. Lateur LM, Van Hoe LR, Van Ghillewe KV, Gryspeerdt SS, Baert AL, Dereymaeker GE (1994) Subtalar coalition: diagnosis with the C sign on lateral radiographs of the ankle. Radiology 193(3):847851

11. Deutsch AL, Resnick D, Campbell G (1982) Computed tomography and bone scintigraphy in the evaluation of tarsal coalition. Radiology 144(1):137-140

12. Sarno RC, Carter BL, Bankoff MS, Semine MC (1984) Computed tomography in tarsal coalition. J Comput Assist Tomogr 8(6):1155-1160

13. Stoskopf CA, Hernandez RJ, Kelikian A, Tachdjian MO, Dias LS (1984) Evaluation of tarsal coalition by computed tomography. J Pediatric Orthop 4(3):365-369

14. Herzenberg JE, Goldner JL, Martinez S, Silverman PM (1986) Computerized tomography of talocalcaneal tarsal coalition: a clinical and anatomic study. Foot Ankle 6(6):273-288

15. Pineda C, Resnick D, Greenway G (1986) Diagnosis of tarsal coalition with computed tomography. Clin Orthop Relat Res 208:282-288

16. Adler SJ, Vannier MW, Gilula LA, Knapp RH (1988) Threedimensional computed tomography of the foot: optimizing the image. Comput Med Imaging Graph 12(1):59-66

17. Emery KH, Bisset GS, Johnson ND, Nunan PJ (1998) Tarsal coalition: a blinded comparison of MRI and CT. Pediatr Radiol 28(8):612-616

18. Nalaboff KM, Schweitzer ME (2008) MRI of tarsal coalition: frequency, distribution, and innovative signs. Bull NYU Hosp Jt Dis 66(1):14-21

19. Patel CV (2009) The foot and ankle: MR imaging of uniquely pediatric disorders. Magn Reson Imaging Clin N Am 17(3):539547

20. Rozansky A, Varley E, Moor M, Wenger DR, Mubarak SJ (2010) A radiologic classification of talocalcaneal coalitions based on $3 \mathrm{D}$ reconstruction. J Child Orthop 4(2):129-135

21. Newman JS, Newberg AH (2000) Congenital tarsal coalition: multimodality evaluation with emphasis on $\mathrm{CT}$ and MR imaging. Radiographics 20(2):321-332

22. Wechsler RJ, Schweitzer ME, Deely DM, Horn BD, Pizzutillo PD (1994) Tarsal coalition: depiction and characterization with CT and MR imaging. Radiology 193(2):447-452 